normal routine of these patients must be disturbed as little as possible, and it is surprising how little it need be.

The determination of the proper time for ophthalmic operations in persons in the declining years of life entails the exercise of considerable judgment, but one must be careful not to err on the too conservative side of the situation lest visual benefit be denied to those who have little else to ease their way along.

\title{
A NOTE ON THE HYDROGEN ION CONCENTRATION OF THE VITREOUS IN EYES AFFECTED BY NAPHTHALENE
}

\author{
BY \\ Dorothy R. Adams and Phyllis Tookey Kerridge \\ UNIVERSITY COLLEGE, LONDON
}

THE second stage of the development of naphthalene cataract in rabbits, is an opacity which begins in the superficial layers of the posterior cortex of the lens, i.e., in the region adjacent to the vitreous. It has been shown (Adams, 1930) that the cataract is almost invariably preceded by the formation of extensive retinal exudates, an oedematous change which might easily affect the vitreous in such a way as to alter its hydrogen ion concentration. Experiments on the isolated lens have demonstrated its sensitivity to the $\mathrm{cH}$ of the surrounding medium. It is known, for instance, that a superficial opacity can readily be produced by immersing a lens in an acid solution, and that by using solutions of different $\mathrm{cH}$ values, one can determine approximately the isoelectric point of the lens (Gullotta, 1926). It was therefore considered worth while to investigate the $\mathrm{cH}$ of the vitreous in rabbits at different times after the administration of naphthalene, in order to see whether it exhibited any change in hydrogen ion concentration which might account for the local condition of the lens, or could be correlated with the oedematous changes in the retina. At the same time it was interesting to find the hydrogen ion concentration of normal vitreous, since it has not been determined previously for the rabbit.

Experimental.-In describing these experiments* it is realised that the values obtained for the hydrogen ion concentration of the

\footnotetext{
*These experiments formed part of the research work for the Committee of Vision
} of the Medical Research Council. 
vitreous post-mortem may not be the same as in vivo, but every effort was made to secure as short an interval as possible between the death of the animal and the measurement of the hydrogen ion concentration of the vitreous of each eye. The average time which elapsed before the first estimation was 6 minutes (see table).

The animals were dosed by means of a stomach tube, with naphthalene dissolved in warm liquid paraffin. In some cases, glucose was given as well, as it seemed to aid absorption and it certainly led to a more rapid appearance of the ocular lesions. (In the table these substances are denoted by $\mathrm{N}$ and $\mathrm{G}$ respectively.)

The ocular lesions included every possible type and were carefully recorded in each case, together with any signs of general toxaemia, although the latter occurred but seldom.

An animal was killed when required by a blow on the head, and the eyeballs were removed rapidly. They were washed free from blood with distilled water, then dried and wrapped in filter paper leaving only the cornea exposed. An incision was made round the equator of the cornea with clean dry scissors. The lens was pushed gently out of the eye, and the posterior lens capsule was perforated. By inverting the eyeball the vitreous was collected without contamination on to a glass slide, whence it was transferred immediately to a glass electrode. It was found advisable to cover the surface of the vitreous with a film of paraffin in order to avoid a slight increase in alkalinity which tended to occur (possibly owing to the loss of $\mathrm{CO}_{2}$ ) if it was left exposed to the air. (See readings on the vitreous of rabbit 22.)

The $\mathrm{pH}$ measurements were made at $18^{\circ} \mathrm{C}$., by the glass electrode method. (Kerridge, 1925-6.)

Results.-Estimations of the $\mathrm{pH}$ of the vitreous of one or of both eyes were made on a series of 16 rabbits which had been dosed previously with naphthalene, and on 3 normals. The results obtained are shown in the table, and may be summarised as follows :-

1. The values for the $\mathrm{pH}$ of the vitreous from 3 normals and from 13 naphthalinised rabbits were within the same range, viz., between $7 \cdot 20$ and $7 \cdot 48$, the average for normals being $7 \cdot 28$, and for the others, 7.31. Samples of vitreous from each of a pair of eyes showed remarkably close agreement in $\mathrm{pH}$. It is interesting to note that the range in $\mathrm{pH}$ of the vitreous at $18^{\circ} \mathrm{C}$. is very close to that of blood at $38^{\circ} \mathrm{C}$. (Austin and Cullen, 1926), although for a strict comparison the $\mathrm{pH}$ of vitreous would have to be determined at $38^{\circ} \mathrm{C}$. 
2 . In the remaining three naphthalinised animals values of $6 \cdot 99,7 \cdot 66$ and $7 \cdot 99$ were obtained without any obvious causative factor.

3. The $\mathrm{pH}$ of the vitreous in naphthalinised animals did not appear to be influenced by any of the following factors :-

(a) The amount of naphthalene given.

(b) The length of time following its administration.

(c) The degree of retinal oedema.

(d) The degree of lens opacity.

(e) Alteration in the physical properties of the vitreous, e.g., colour and consistency.

$(f)$ Symptoms of general toxaemia.

4. Normal vitreous shows an increase in alkalinity on being left exposed to air (see rabbit 22). If it is covered with a film of paraffin, successive readings on the same sample are remarkably constant (see rabbits $25,23,24$, and 20 ).

5. In many cases the vitreous was yellow and fluid, while the lenses from the same eyes turned golden yellow on being left exposed to air.

6. The vitreous from an eye which contained deposits of crystals gave a normal $\mathrm{pH}$ value (see rabbit 15. )

Conclusion.-The hydrogen ion concentration of the vitreous does not appear to change at any moment after the administration of naphthalene in such a way as to account for the development of cataract, neither is it affected by other intra-ocular changes such as retinal oedema or atrophy.

\section{REFERENCES}

Adams. - The nature of the ocular lesions produced experimentally by naphthalene. Brit. Jl. of Ophthal., Vol. XIV, p. 49, 1930.

Austin and Cullen.-The hydrogen ion concentration of the blood in health and disease. Medicine Monographs, Vol. VIII, Chap. II, 1926.

Gullotta.-Il punto isoelettrico delle proteine della lente cristallina. Arch. di Scienza Biol., Vol. VIII, p. 48, 1926.

Kerridge, P. T. -The use of the glass electrode in biochemistry. Biochem. Jl., Vol. XIX, p. 611, 1925.

- The use of glass electrodes. Jl. Sci. Instruments, Vol. III, p. 404, 1925-6. 


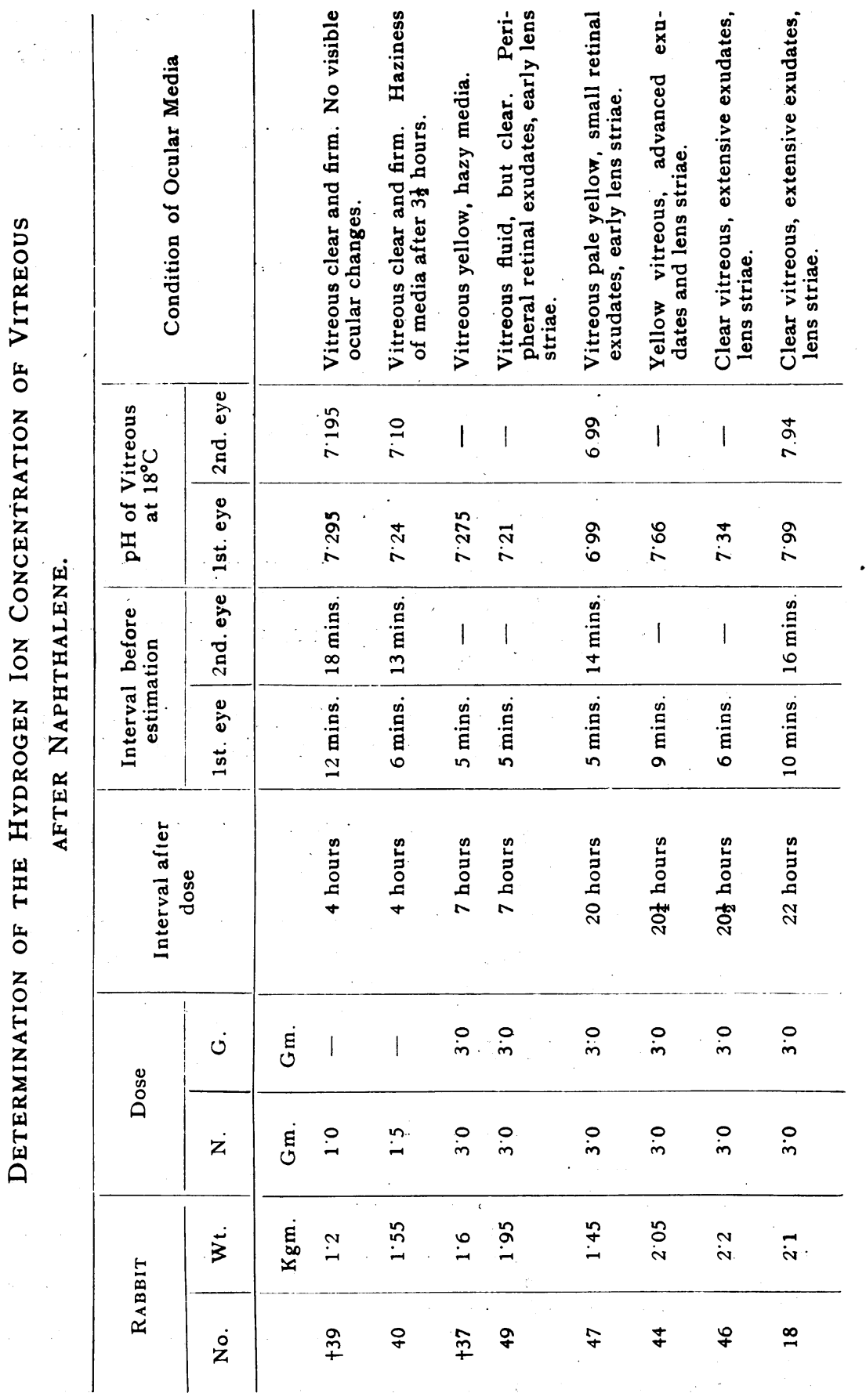




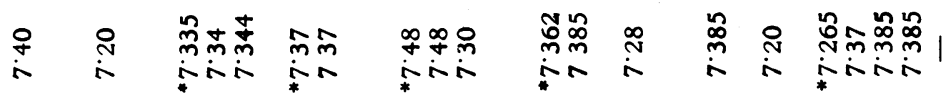

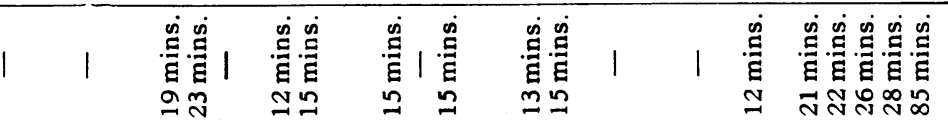

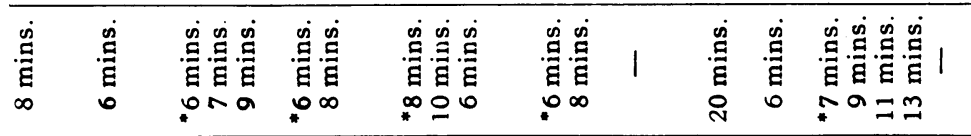

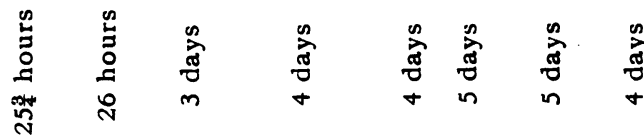

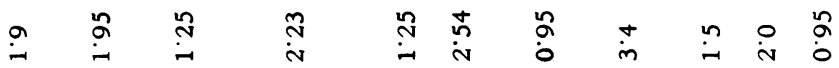

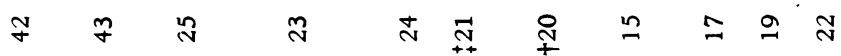

